\title{
Stage I T Lymphoblastic Leukemia/Lymphoma
}

National Cancer Institute

\section{Source}

National Cancer Institute. Stage I T Lymphoblastic Leukemia/Lymphoma. NCI

Thesaurus. Code C8697.

Ann Arbor Classification: Stage I: Involvement of a single lymph node region (I); or localized involvement of a single extralymphatic organ or site in the absence of any lymph node involvement (IE) (Rare in Hodgkin lymphoma). - 2003 